\title{
Avatars: The Other Side of Proteus's Mirror
}

Charly Harbord

Euan W. Dempster

This is a post-peer-review, pre-copyedit version of an article published in the Proceedings of Entertainment Computing and Serious Games. First IFIP TC 14 Joint International Conference on Entertainment Computing and Serious Games.

The final authenticated version is available online at: https://doi.org/10.1007/978-3-030-34644-7 37 


\title{
Avatars: the other side of Proteus's Mirror
}

\author{
A study into Avatar choice regarding perception. \\ Charly Harbord [0000-0001-7079-852X] \\ ${ }^{1}$ Abertay University, Bell Street, Dundee, DD1 1HG \\ c-a.harbordeabertay.ac.uk
}

\begin{abstract}
The trend for online interactions, can be regarded as being 'anti-socially social', meaning that a great deal of time is spent playing, working and socializing with the internet serving as the communication conduit. Within that Virtual Social Environment very deep relationships are formed and maintained without the parties ever having met each other face-to-face. Raising the question how much does the physical appearance of an avatar influence the perception of the person behind it? Are relationships informed by appearance even in the virtual world and what implications does that have for second language acquisition? This paper leads to a small-scale research project where a selection of avatars with various racially identifiable characteristics were used to identify which avatars a second language speaker would feel more at ease interacting with in the target language. The resultant research aims to test three hypotheses regarding preferred avatar choice for second language users based solely on perceptions.
\end{abstract}

Keywords: Avatars, Second Language Acquisition, Proteus Effect, Perception, Chinese Concept of Face, RPGs

\section{$1 \quad$ Literature Study:}

\subsection{Proteus Mirror}

Yee and Bailenson (13) coined the term 'The Proteus Effect' to define the way in which in-game behaviors are influenced by the appearance of a player's avatar. These selfperceptions and resulting actions can be seen as independent from perceptions of other players. It is important to the future planned research on RPGs and second language acquisition to ascertain whether interaction with other avatars is based on cultural aesthetics and perceived personality. This paper aims to look through the other side of Proteus's mirror to examine what the viewer sees and how that affects their online ingame interactions. 


\subsection{Avatars}

Avatars are endemic throughout online communities and modern culture (1). High levels of personalization allow players to select exactly how they wish to present themselves and therefore how they wish to be perceived by others; creating a virtual mask $(2 ; 4 ; 3)$. When a new avatar is encountered, impulse judgements are often made regarding the personality of the user informing the type of interpersonal relationship that may be formed and acting a predictor of the behavioral profile of the user; known as the 'Thin-Slice Effect' (10). The high level of avatar customization freely available now to users, means that the thin-slice style of judgement has greater validity (4). However, the 'thin-slice' may be open to a certain level of bias with the viewer superimposing how honestly, they themselves self-represent as an avatar. The study aims to investigate how avatar perception affects relationships in general and specifically as second language interlocutors. It is intended that the focus of this small-scale research will be purely on the aesthetics of avatar perception and the level of subjectivity held by the viewer towards the visual cues.

\subsection{Face and Second Language Acquisition}

When relationships are formed and maintained entirely online via the medium of avatars, the player is likely to feel less anxiety due to the 'faceless' anonymity of the interaction. Additionally, the lack of visible social prompts based on context that may otherwise cause interference, allow for a freer interaction without anxiety or inhibition (8). This is particularly prevalent cross-linguistically between English and Mandarin. The use of a racially neutral avatar can help to alleviate the anxiety that can be felt when communicating with a native speaker in the target language (7). The concept of 'Face' resonates quite strongly with Chinese native speakers $(9 ; 11)$. Face is intrinsic to Chinese culture; it holds that the perception of a person and how they present themselves is potentially more important than the actual reality. Avatars allow a degree of freedom within the interactions as there is no perceived loss of face (11). Feeding into the negation of face is a sense of impermanence and reduction of responsibility with avatar relationships; should a major mistake or social faux pas happen instead of trying to make amends a new avatar can be created, and the cycle starts over (5).

\section{Avatar Small Scale Study}

The main area of research in the small-scale study was directed at second language speakers and how willing or not they are to interact with avatars that represent the cultural and racial background of the target language. Participants were shown a selection of avatars representing a potential target language interlocutor. They were then asked to express what their perception of the character is and what level of interaction they would take. 
It was predicted that the choice of avatar for in game interactions would mimic those in real life; that the second language speakers would feel more at ease interacting in the target language with a more culturally neutral character. Within the classroom environment students are quite happy to use the target language to converse and feel less pressure to be perfect at the language, thereby creating a rapport and a safe space with peers of similar ability (9). However, when faced with a native speaker, students reported feeling anxious and reluctant to interact in the target language (9). It was predicted that players would feel more comfortable interacting in the second language if their avatar looks more culturally representative to the target language and the interlocutor's avatar looks more generic.

In order to test the hypothesis as a proof of concept, 16 avatars were designed to represent multiple racial identifiers and fantasy styles (see figure 1). The participants were 20 Chinese students who have English as a second language and 20 home students who learn Mandarin. They were asked to select which of the avatars they would choose in three different scenarios. The scenarios are based on the participant being a second language user interacting with native speakers in the target language in an RPG. 1) Which avatar that they would feel comfortable interacting with in the target language with. 2) Which avatar that they would feel uncomfortable speaking in the target language with. 3) Which avatar would they choose for themselves as the second language speaker. The preferred avatars will inform the choices given in the resultant RPG that is being created by the author for the larger scale research project into the use of RPG for the mutual enhancement of both Mandarin and English.
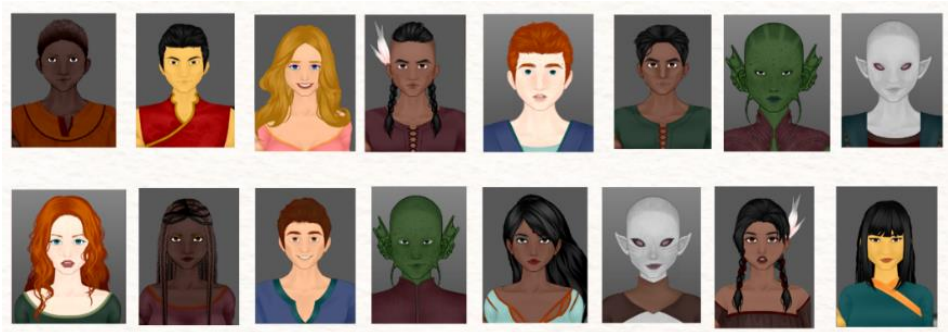

Figure 1: Avatars designed for the study.

Hypothesis 1: Participants are more likely to choose an avatar that resembles a native speaker. Players will select an avatar to look more racially appropriate to the target language and be more comfortable interacting with avatars that present as less racially stereotypical.

Hypothesis 2: Participants are more likely to be comfortable interacting with a more racially neutral character. Avatars with a lower level of racial and cultural identifiers act as the interlocutor helps to negate the levels of anxiety that a second language speaker may have when interacting with a native speaker.

Hypothesis 3: Participants are more likely to experience increase lack of inhibition and increased motivation due the anonymity of using an avatar. Avatars enhance not only the immersion and emotional investment also the role-play and communication 
between players (6). The use of an avatar can help negate the anxiety that a learner may feel within face-to-face conversation in the target language (8).

\section{Preliminary Results:}

The first round of testing was the Chinese participants carried out via an anonymous online questionnaire.
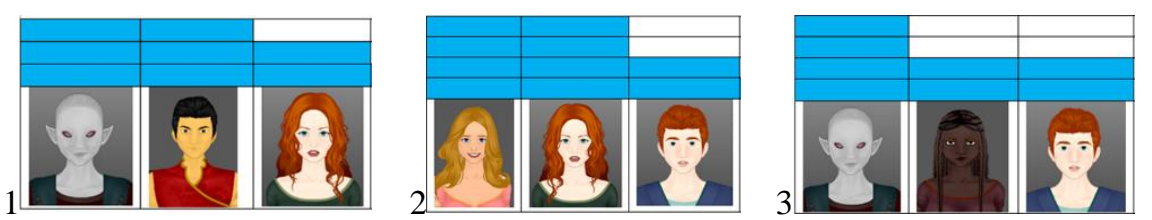

1 shows the avatars that the Chinese participants were most likely to choose to represent themselves. The reasons given for these choices were: "the Chinese boy looks most like me" "this avatar is the most neutral" and "she is really pretty so more people may choose to talk to me".

2 shows the avatars that the Chinese participants would choose to interact with in the target language. The reasons given for these selections were "She is the prettiest", "I like her smile", "She looks Scottish and I want to go to Scotland".

3 shows the avatars that the Chinese participants would not want to interact with. The reasons given for these selections were "This avatar is too ugly", "I don't like it's face".

Whilst the chosen avatars for each scenario reflected the author's predictions the reasonings veered from the original hypotheses. The Chinese participants demonstrated aesthetics to be the main driving force behind their choices. When considered with the burgeoning "meinü jingji, beauty economy" (12) in China the justifications are not surprising. Closely linked to face; beauty is intrinsically connected to success and prestige.

\section{Conclusion}

The results have allowed the avatar choice given to the Chinese players to be narrowed down to the top three from $1 \& 2$. The home participants questionnaire will be carried out in September 2019; informing the avatar choices given to players of the finalized RPG for the larger study. Further research will focus on the investigating the socio-cultural implications of avatar choice and how aesthetics of perception affects player interactions in the target language. Additionally, connections will be drawn between the historical culture of mask use and the Chinese concept of 'Face'. 


\section{References:}

1. Dunn, R. and Guadagno, R. (2012). My avatar and me - Gender and personality predictors of avatar-self discrepancy. Computers in Human Behavior, [online] 28(1), pp.97-106. Available at: http://dx.doi.org/10.1016/j.chb.2011.08.015 [Accessed 29 May 2019].

2. Leding, J., Horton, J. and Wootan, S. (2015). The contrast effect with avatars. Computers in Human Behavior, [online] 44, pp.118-123. Available at: http://dx.doi.org/10.1016/j.chb.2014.11.054 [Accessed 29 May 2019].

3. Li, D., Liau, A. and Khoo, A. (2013). Player-Avatar Identification in video gaming: Concept and measurement. Computers in Human Behavior, [online] 29(1), pp.257-263. Available at: http://dx.doi.org/10.1016/j.chb.2012.09.002 [Accessed 29 May 2019].

4. Lin, H. and Wang, H. (2014). Avatar creation in virtual worlds: Behaviors and motivations. Computers in Human Behavior, [online] 34, pp.213-218. Available at: http://dx.doi.org/10.1016/j.chb.2013.10.005 [Accessed 29 May 2019].

5. Nylund, A. and Landfors, O. (2015). Frustration and its effect on immersion in games. Masters. Umea University.

6. Peterson, M. (2006). Learner interaction management in an avatar and chat-based virtual world. Computer Assisted Language Learning, [online] 19(1), pp.79-103. Available at: http://dx.doi.org/10.1080/09588220600804087 [Accessed 24 Jan. 2019].

7. Peterson, M. (2011). Digital gaming and second language development: Japanese learners interactions in a MMORPG. Digital Culture and Education, 3(1), pp.56-73.

8. Rankin, Y. A., McNeal, M., Shute, M. W., \& Gooch, B. (2008). User centered game design: evaluating massive multiplayer online role playing games for second language acquisition. In Proceedings of the 2008 ACM SIGGRAPH symposium on video games (pp. 43-49). ACM.

9. Rublik, N. (2018). Chinese Cultural Beliefs: Implications for the Chinese Learner of English. Sino-US English Teaching, [online] 15(4). Available at: http://dx.doi.org/10.17265/15398072/2018.04/001 [accessed 4 Jun. 2019]

10. Shin, M., Kim, S. and Biocca, F. (2019). The uncanny valley: No need for any further judgments when an avatar looks eerie. Computers in Human Behavior, [online] 94, pp.100-109. Available at: http://dx.doi.org/10.1016/j.chb.2019.01.016 [Accessed 27 May 2019].

11. Wen, W. and Clement, R. (2003). A Chinese Conceptualisation of Willingness to Communicate in ESL. Language, Culture and Curriculum, [online] 16(1), pp.18-38. Available at: http://dx.doi.org/10.1080/07908310308666654 [Accessed 30 May 2019].

12. Xu, G. and Feiner, S. (2007), Meinu Jingii/China's beauty economy: Buying looks, shifting value, and changing place. Feminist Economics, [online]13(3-4), pp.307-323. Available at: http://dx.doi.org/10.1080/13545700701439499 [Accessed on 21 Jun. 2019]

13. Yee, N. and Bailenson, J. (2007). The Proteus Effect: The Effect of Transformed Self-Representation on Behavior. Human Communication Research, [online] 33(3), pp.271-290. Available at: http://dx.doi.org/10.1111/j.1468-2958.2007.00299.x [Accessed 23 Jan. 2019] 